OPEN ACCESS

Edited by:

Antonio Francesco Logrieco, Italian National Research Council

(CNR), Italy

Reviewed by:

Shihua Wang,

Fujian Agriculture and Forestry

University, China

Shizhu Zhang,

Nanjing Normal University, China

*Correspondence:

Yang Liu

liuyang01@caas.cn

Specialty section: This article was submitted to

Food Microbiology,

a section of the journal

Frontiers in Microbiology

Received: 04 July 2019 Accepted: 12 November 2019

Published: 28 November 2019

Citation:

Wang G, Zhang H, Wang Y, Liu F, Li E, Ma J, Yang $B$, Zhang $C$, Li $L$ and Liu Y (2019) Requirement of LaeA,

$V e A$, and VelB on Asexual

Development, Ochratoxin A

Biosynthesis, and Fungal Virulence

in Aspergillus ochraceus.

Front. Microbiol. 10:2759.

doi: 10.3389/fmicb.2019.02759

\section{Requirement of LaeA, VeA, and VelB on Asexual Development, Ochratoxin A Biosynthesis, and Fungal Virulence in Aspergillus ochraceus}

\author{
Gang Wang ${ }^{1}$, Haiyong Zhang ${ }^{1}$, Yulong Wang ${ }^{1}$, Fei Liu' ${ }^{1}$, Erfeng Li $^{2}$, Junning Ma ${ }^{1}$, \\ Bolei Yang ${ }^{1}$, Chenxi Zhang ${ }^{1}$, Li Li ${ }^{1}$ and Yang Liu ${ }^{1 *}$
}

${ }^{1}$ Key Laboratory of Agro-products Quality and Safety Control in Storage and Transport Process, Ministry of Agriculture and Rural Affairs, Institute of Food Science and Technology, Chinese Academy of Agricultural Sciences, Beijing, China,

${ }^{2}$ Horticulture and Landscape College, Tianiin Agricultural University, Tianjin, China

Aspergillus ochraceus is reported to be the major contributor of ochratoxin A (OTA), classified as one of the possible human carcinogen (group 2B) by the International Agency for Research on Cancer. The heterotrimeric velvet complex proteins, LaeANeANelB, have been most studied in fungi to clarify the relation between light-dependent morphology and secondary metabolism. To explore possible genetic targets to control OTA contamination, we have identified laeA, veA, and velB in $A$. ochraceus. The loss of laeA, $v e A$, and velB yielded mutants with differences in vegetative growth and conidial production. Especially, $\Delta$ laeA almost lost the ability to generate conidiaphore under dark condition. The deletion of laeA, veA, and velB drastically reduced the production of OTA. The wildtype $A$. ochraceus produced about 1 and $7 \mu \mathrm{g} / \mathrm{cm}^{2}$ OTA under light and dark conditions on media, whereas the three gene deletion mutants produced less than $20 \mathrm{ng} / \mathrm{cm}^{2}$ OTA, which was correlated with a down regulation of OTA biosynthetic genes. Pathogenicity studies of $\Delta$ laeA, $\Delta v e A$, and $\Delta$ velB showed their reduction in disease severity in pears. Furthermore, $66.1 \%$ of the backbone genes in secondary metabolite gene cluster were significantly regulated, among which $81.6 \%$ were downregulated. Taking together, these results revealed that velvet complex proteins played crucial roles in asexual development, secondary metabolism, and fungal virulence in A. ochraceus.

Keywords: Aspergillus ochraceus, ochratoxin A, LaeA, VeA, VelB, secondary metabolism, development, virulence

\section{INTRODUCTION}

Ochratoxin A (OTA) is the secondary metabolite of Aspergillus and Penicillium species (Wang et al., 2016a,b). That poses a serious health hazard according to its mycotoxic properties (Taniwaki et al., 2018). It is classified as a possible human carcinogen (group 2B) by the International Agency for Research on Cancer (IARC, 1993). OTA was first isolated from A. ochraceus in 1965 (van der Merwe et al., 1965). And it was reported to be the major contributor of OTA in cereal, Zea mays, coffee, fruits, and beverage (Mantle, 2002). 
The biosynthetic pathway of OTA has been extensively studied in the past decades (William and Hamilton, 1979; Wang et al., 2015; Gallo et al., 2017; Geisen et al., 2018). Wang has identified a conserved OTA biosynthetic gene cluster by comparatively analysis of six OTA-producing fungi and clarified its biosynthetic pathway by deletion mutants of four structural genes (otaA, $B, C$, and $D$ ) and one regulatory gene (otaR1) (Wang et al., 2018a,b). Environmental factors are crucial to regulation of OTA production (Selouane et al., 2009; Abarca et al., 2019). The mechanism of OTA biosynthesis is very complex and acts at different levels. Generally, environmental signals transmit to biosynthetic cluster to activate/repress the production of OTA by global regulators and multiprotein complexes. For example, Aoyap1, a transcription factor related to oxidative stress, regulated OTA synthesis by controlling cell redox balance in A. ochraceus (Reverberi et al., 2012). The transcriptional factors AopacC (Wang et al., 2018a,b) and hog (Schmidt-Heydt et al., 2012) that are functionally performed $\mathrm{pH}$ signaling and osmotic stress were also involved in the regulatory mechanism of OTA biosynthesis at $\mathrm{pH}$ stress and osmotic stress, respectively. The heterotrimeric velvet complex, VelB/VeA/LaeA, has been most studied in fungi to clarify the relation between light-dependent morphology and secondary metabolism. In A. nidulans, VeA bridges VelB to LaeA, the nuclear master regulator of secondary metabolism (Bayram et al., 2008). LaeA has also been suggested as an epigenetic regulator for its methyltransferase functions toward amino acid lysine and arginine. Several structure homologous LaeA proteins have been identified in A. fumigatus (Bok et al., 2005), A. oryzae (Oda et al., 2011), Cochliobolus heterostrophus (Wu et al., 2012), Fusarium oxysporum (Lopez-Berges et al., 2014), P. chrysogenum (Veiga et al., 2012), and Trichoderma reesei (KarimiAghcheh et al., 2013) and demonstrated profound influence on sporulation capacity, mycelial growth, sclerotia formation, and secondary metabolite production.

Several studies have been conducted to regulate mycotoxin biosynthesis by LaeA. The deletion of laeA in A. flavus led to the loss of aflatoxin mediated by the expression loss of aflR, specific transcription factor in aflatoxin biosynthetic cluster. The conidial production, sclerotia formation, and host colonization were repressed in the $\Delta$ laeA of A. flavus (Kale et al., 2008). Deletion of laeA and $v e A$ greatly reduced sporulation and strongly copromised the alternariol and alternariol monomethyl ether production (Estiarte et al., 2016). In A. carbonarius, Crespo-Sempere suggested that VeA and LaeA have an important role regulating conidiation and OTA biosynthesis (Crespo-Sempere et al., 2013). The veA gene was proven to act as a positive regulator of conidia production, OTA biosynthesis, and oxidative stress tolerance in $A$. niger (Zhang et al., 2018). A. steynii, A. niger, P. nordicum, and $P$. verrucosum were described about their ability to produce OTA response to light (Schmidt-Heydt et al., 2010, 2011). However, comprehensive study about velvet complex regulated OTA biosynthesis responding to light is needed.

There is still limited information regarding to the link of light and OTA biosynthesis and their regulatory mechanism in A. ochraceus, except Aziz reported white and UV light affected mycelial growth and OTA production in 1997 (Aziz and Moussa, 1997). Nothing has been reported about the function of velvet complex proteins in A. ochraceus. For this purpose, we have identified and deleted the members of velvet complex (laeA, veA, and velB) in A. ochraceus and explored their regulatory role in growth morphology, OTA biosynthesis and fungal virulence on pears. Furthermore, we demonstrated how LaeA affects secondary metabolism in A. ochraceus at gene expression level.

\section{MATERIALS AND METHODS}

\section{Strains and Growth Conditions}

The wild type (WT) strain A. ochraceus fc-1 used in this study was isolated, characterized, and genome sequenced in our laboratory (Wang et al., 2018a,b). WT and mutant strains were routinely cultured at $28^{\circ} \mathrm{C}$ under dark condition. For phenotype and gene expression studies, all utilized strains were cultured on potato dextrose agar (PDA, BD DifcoTM, USA) at $28^{\circ} \mathrm{C}$. Each strain was cultured on four plates as technical replicates, and each experiment was repeated three times as biological replicates.

\section{Phylogenetic Tree and Functional Analysis}

LaeA, VeA, and VelB amino acid sequences from A. nidulans (Bayram et al., 2008), A. flavus (Kale et al., 2008) and Cochliobolus heterostrophus (Wu et al., 2012) were used as queries, and basic local alignment search tool algorithm was used to search LaeA, VeA, and VelB from the genome of A. ochraceus, A. niger, A. welwitschiae, A. lacticoffeatus, A. sclerotioniger, A. steynii, and $P$. nordicum from the National Center for Biotechnology Information resources (NCBI). The amino acid sequences of LaeA were aligned by MUSCLE, and a maximum likelihood phylogeny was constructed by treeBeST using 1,000 bootstrap replicates.

\section{Generation of Gene Deletion Mutants}

To construct laeA, veA, and velB mutants, previous approach reported in our group was used, and the deletion cassettes were generated by overlap PCR procedures (Wang et al., 2018a,b). Primers utilized in this study were listed in Supplementary Table S1. And then fusion PCR products were transformed into the protoplasts of $A$. ochraceus. Transformants were verified by Southern blotting. Briefly, approximately $20 \mu \mathrm{g}$ genomic DNA of each sample was complete-digested and separated $1 \%$ agarose gel and transferred to a Hybond-N+ nylon membrane (GE healthcare, UK). After alkali denaturation and neutralization, hybridization was detected with digoxigenin-labeled probes using DIG high-prime DNA labeling and detection starter Kit II (Roche, Basel, Switzerland) according to the instructions of the manufacturer. Primers for probe amplification were listed in Supplementary Table S1.

\section{Phenotypic Studies of Mutants}

For mutant's growth assessment, PDA plates were inoculated at center with $1 \mu \mathrm{l}$ of conidia suspension $\left(10^{6}\right.$ conidia/ml $)$ of 
each strain and cultures were incubated at $28^{\circ} \mathrm{C}$ for 9 days under two conditions, white light (Mazda, $23 \mathrm{~W}$ CFT/827, $1,485 \mathrm{~lm}$ ) and darkness. The growth rate was analyzed by measuring the colony diameter of each mutant. For phenotypic study, the hyphae and spores were observed under optical microscope and electron microscope. For further analysis, conidia were collected from six agar plugs $(1 \mathrm{~cm}$ diameter) from equivalent zones of fungal surface of PDA. The collected samples were homogenized and diluted in $0.1 \%$ Tween- 80 and counted by a hemocytometer.

\section{Analysis of Ochratoxin A Production}

For the investigation of OTA, WT, $\Delta$ laeA, $\Delta$ veA, and $\Delta$ velB of $A$. ochraceus were cultured on PDA for 9 days under light and dark conditions. Six agar plugs $(1 \mathrm{~cm}$ diameter $)$ from equivalent zones of fungal surface of PDA were collected and extracted with $6 \mathrm{ml}$ methanol ultrasonically. Then, the supernatant was filtered through a $0.22 \mu \mathrm{m}$ filter into a vial. Next, HPLC analysis was performed on an Agilent HPLC system for analyzing the concentration of OTA as previously described method (Wang et al., 2018a,b).

\section{Pathogenicity Assay}

Fresh pears (Pyrus $\times$ bretschneideri) were selected to test the pathogenicity of WT and mutant strains of A. ochraceus in vitro. The upper surface of pears were disinfected three times with $0.1 \%$ sodium hypochlorite $(\mathrm{NaClO})$ for $10 \mathrm{~s}$ and rinsed with sterilized water for $30 \mathrm{~s}$. Each pear was punctured by sterilized needle to approximately $2 \mathrm{~mm}$ depth to make a wound $(2 \mathrm{~mm}$ diameter) for inoculation, injected $2 \mu$ l conidia suspension $\left(10^{6}\right.$ conidia $\left./ \mathrm{ml}\right)$ in wound, in contrast sterilized water was served as control and incubated at $28^{\circ} \mathrm{C}$ under dark condition. The diameter of scab was measured after 5 and 9 days.

\section{DNA and RNA Isolation}

The mycelium of $A$. ochraceus strains were harvested via filtration. Genomic DNA was isolated using a Qiagen DNeasy kit, according to the manufacturer's protocol. For RNA isolation, the A. ochraceus mycelium tissues were grown on PDA medium at $28^{\circ} \mathrm{C}$ for 9 days under light condition. RNA was extracted using TRIZOL reagent (Invitrogen, USA) following the manufacturer's protocol.

\section{Real-Time Polymerase Chain Reaction Analysis and Quantitative Real-Time Polymerase Chain Reaction Analysis}

Three biological replicates were performed for each analysis of the relative expression levels. Reverse transcription of $500 \mathrm{ng}$ RNA was performed with a TIANScript II RT Kit (TIANGEN, China). The A. ochraceus gadph gene served as an internal standard. Primers for the RT-PCR amplification were listed in Supplementary Table S2. The cDNA was analyzed by qRT-PCR using SYBR Premix Ex Taq $^{\mathrm{TM}}$ II (TAKARA) on a BIO-RAD CFX96 (BIO-RAD). The gadph gene serving as house-keeping gene was used for normalization. The relative expression values were calculated and the expression ratios were quantified using the $2^{-\Delta \Delta C t}$ method. Primers were listed in Supplementary Table S3.

\section{Statistical Analysis}

All data were analyzed with IBM SPSS statistics version 20 and presented with the means and standard deviation. The statistical significances among sample groups were calculated with ANOVA and means were compared by least significant difference (LSD) and Duncan's test. The difference was regard to be statistic significant at $p<0.05$.

\section{RESULTS}

\section{Identification, Analysis, and Disruption of LaeA, VeA, and VelB in A. ochraceus}

In order to identify velvet protein homologs in $A$. ochraceus, the genome sequence of $A$. ochraceus was interrogated using Blast alignment approach. BlastP searches were performed using LaeA, VeA, and VelB amino acid sequences from A. nidulans, A. flavus and Cochliobolus heterostrophus as the probes and the homologs AoFC_03061, AoFC_07220 and AoFC_09406 were identified. LaeA from A. steynii (XP_024703593.1), VeA from A. tanneri (THC96327.1), and VelB from A. tanneri (THC97134.1) were found to be most related to velvet complex proteins in A. ochraceus, with the identity of 95.2, 72.5 and $89.6 \%$, respectively. A phylogenetic tree of evolutionary relationship of LaeA proteins from various species including OTA producing fungi was constructed (Figure 1), revealing that LaeA was conserved among the Aspergillus species. Inactivation of LaeA, VeA, and VelB locus was obtained by homologous replacement of the genes by encoding gene of hygromycin B phosphotransferase (hygR). The strategy of mutant generation was shown in Supplementary Figure S1A. The isolate resistant to hygromycin B was screened by PCR using primers in marker gene namely hygR and outside the knockout cassette (Supplementary Figure S1B). At least three transformants of each gene disrupted mutant were obtained from the mutant generation. Southern blot analysis also showed that $\Delta$ laeA (Figure 2A), $\Delta v e A$ (Figure 2B), and $\Delta v e l B$ (Figure 2C) lack the target genes (laeA, veA, and velB).

\section{Involvement of LaeA, VeA, and VelB in Asexual Development, Growth Rate, and Conidiation}

A series of difference related to colony morphology, asexual development and conidiation were observed in $\Delta$ laeA, $\Delta$ veA, and $\Delta$ velB compared with the WT of $A$. ochraceus on PDA media under light and dark conditions. Under light condition as shown in Figure 3A, the WT colonies grew in yellow uniform layer while the laeA deletion mutant grew as a white-yellow cover. We also observed a pigment reduction for $\Delta$ laeA, and a pigment increasing for $\Delta \mathrm{veA}$ and $\Delta \mathrm{velB}$ in the back of the Petri dishes. Under dark condition, the WT A. ochraceus showed more pigmentation compared to the light condition. The $\Delta$ laeA grew as a white color for the decrease of spores and pigment (Figure 3A). A reduction of conidiophore in $\Delta$ laeA compared with the other strains from the colony edge under dark condition by scanning electron micrograph was observed (Figure 3B). 


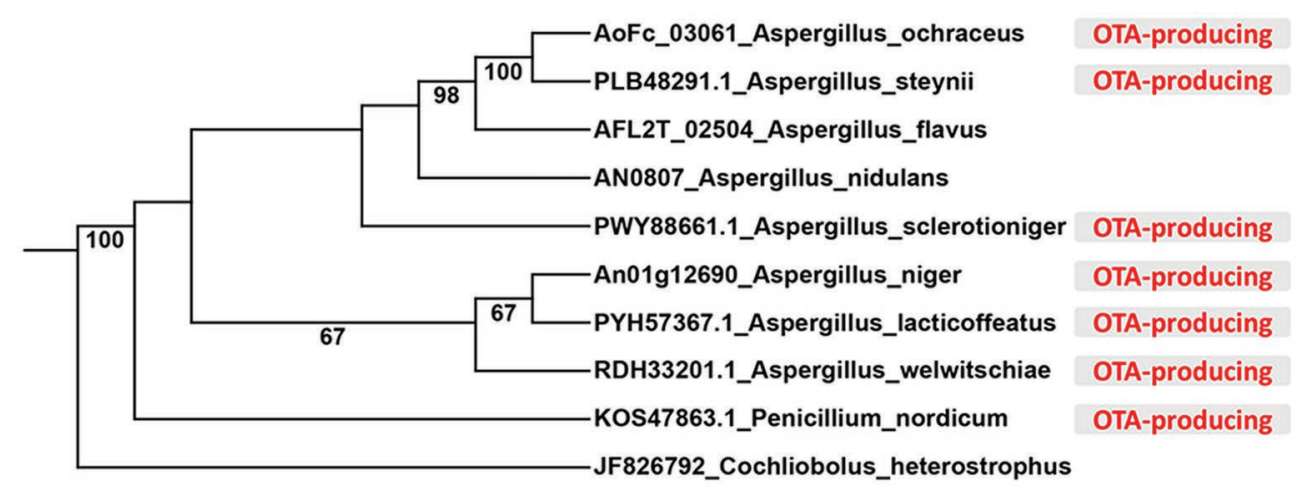

FIGURE 1 | Phylogenetic relationship of LaeA protein from different species. The OTA-producing fungi were marked in red color.

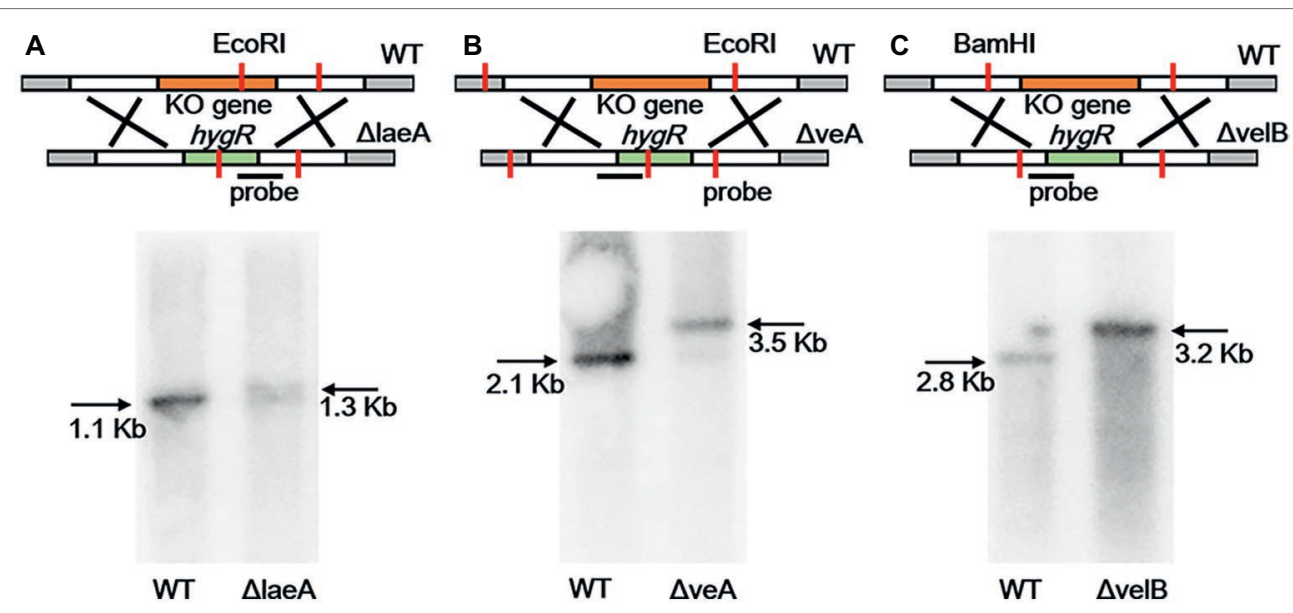

FIGURE 2 | Southern blotting verification of laeA, veA, and velB gene deletion. (A) The WT and $\triangle$ laeA isolates were digested with EcoRI. A fragment amplified from $\Delta$ laeA was used as the probe. (B) The WT and $\Delta$ veA isolates were digested with EcoRI. A fragment amplified from $\Delta v e A$ was used as the probe. (C) The WT and $\Delta$ velB isolates were digested with BamHI. A fragment amplified from $\Delta$ velB was used as the probe.

Light condition had no effect on the growth rate of $A$. ochraceus strains for WT and $\Delta$ laeA, while repressing the growth of $\Delta$ veA and $\Delta$ velB $(p<0.05)$. The growth rate was significantly decreased in $\Delta$ laeA, $\Delta$ veA and $\Delta$ velB compared with the WT (Figure 4A). Mycotoxin-producing fungi caused extensive infestations by generating asexual spores called conidiaspore. To investigate the involvement of LaeA, VeA and VelB in conidiation, the conidiaspore number was counted for strains cultured for 9 days under light and dark conditions. We found conidial generation was increased in the light condition for the A. ochraceus strains, although the conidiaspore amount of $\Delta$ velB under light and dark condition demonstrated non-significant difference at statistic level (Figure 4B). The deletion of laeA resulted in a drastic reduction of conidial generation, whose inactivation leading to A. ochraceus almost loss the ability to generate conidiaspore under dark condition (Figure 4B). The conidiaphore amount of $\Delta$ laeA and $\Delta$ velB under light condition demonstrated significant difference compared with the WT. These results indicated the velvet complex proteins (LaeA, VeA and VelB) play important roles in colony phenotype, growth rate and conidiation.

\section{Requirement of LaeA, VeA, and VelB in Ochratoxin A Biosynthesis}

In order to investigate whether LaeA, VeA, or VelB is linked to secondary metabolism related to OTA biosynthesis, the crude extractions of $A$. ochraceus of 9-day-old cultures were analyzed by HPLC. The results showed the deletion of laeA, veA, and $v e l B$ drastically reduced the production of OTA. The WT $A$. ochraceus produced about land $7 \mu \mathrm{g} / \mathrm{cm}^{2}$ OTA under light and dark condition on media, while the three gene deletion mutants produced less than $20 \mathrm{ng} / \mathrm{cm}^{2}$ OTA (Figure 5A). We observed white light was an inhibitory factor for OTA biosynthesis. To further elucidate the function of LaeA as regulator of OTA biosynthesis, the expression level of genes in the OTA biosynthetic cluster was comparatively examined in WT and $\Delta$ laeA in the dark condition. As shown in Figure 5B, the results of qRT-PCR analysis confirmed the expression level of otaA, otaB, otaC, otaR1, and otaD was downregulated 2-40fold in $\Delta$ laeA compared to those genes in WT. The upstream gene AoFC_09697 and downstream gene AoFC_09703 showed different expression profiles in WT and also $\Delta$ laeA with respect 


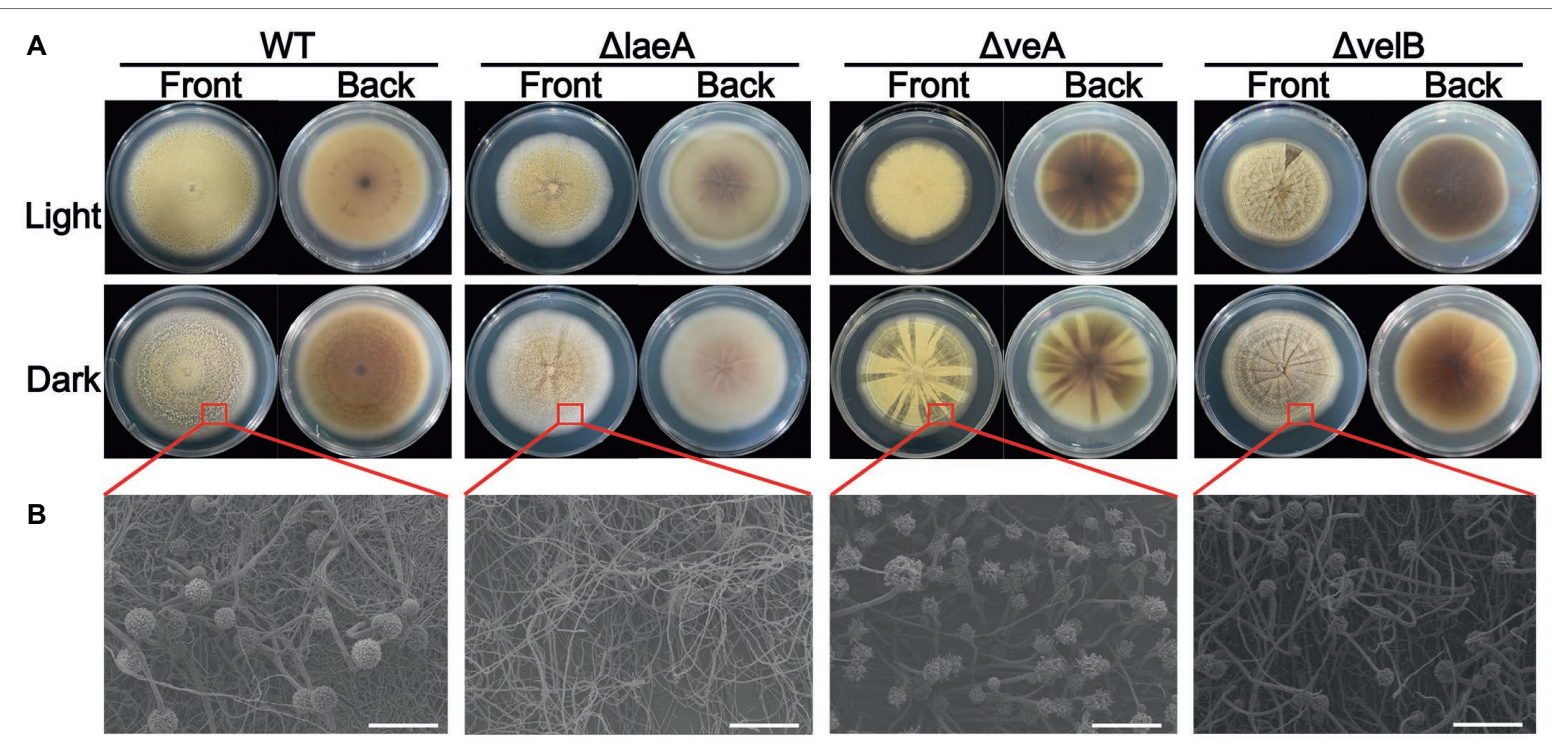

FIGURE 3 | Colony view of the WT, $\Delta$ laeA, $\Delta$ veA, and $\Delta$ velB strains of $A$. ochraceus. (A) The front and back of $A$. ochraceus colony under light and dark conditions. (B) Scanning electron micrograph of $A$. ochraceus strains (scale bar $=200 \mu \mathrm{m}$ ). The red box represented the part of colony for observation.

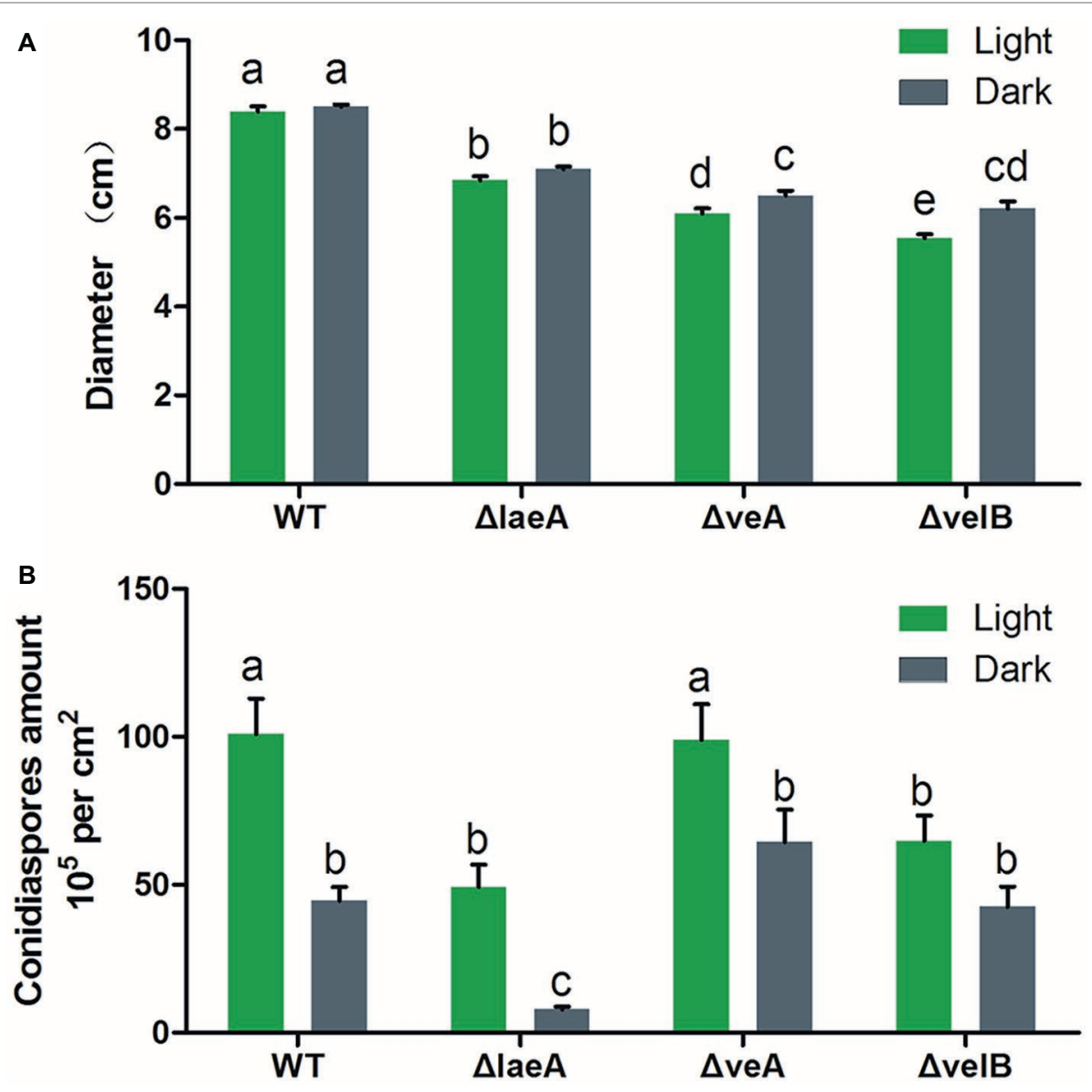

FIGURE 4 I Effect of LaeA, VeA, and VelB deletion on the colony growth and conidiation of $A$. ochraceus. (A) Diameter of WT, $\Delta$ laeA, $\Delta$ veA, and $\Delta$ velB under light and dark conditions. (B) Conidiaspore production of WT, $\Delta$ laeA, $\Delta$ veA, and $\Delta$ velB under light and dark conditions. Different letters indicate a significant difference between the corresponding values $(p<0.05)$ with three biological replicates. 


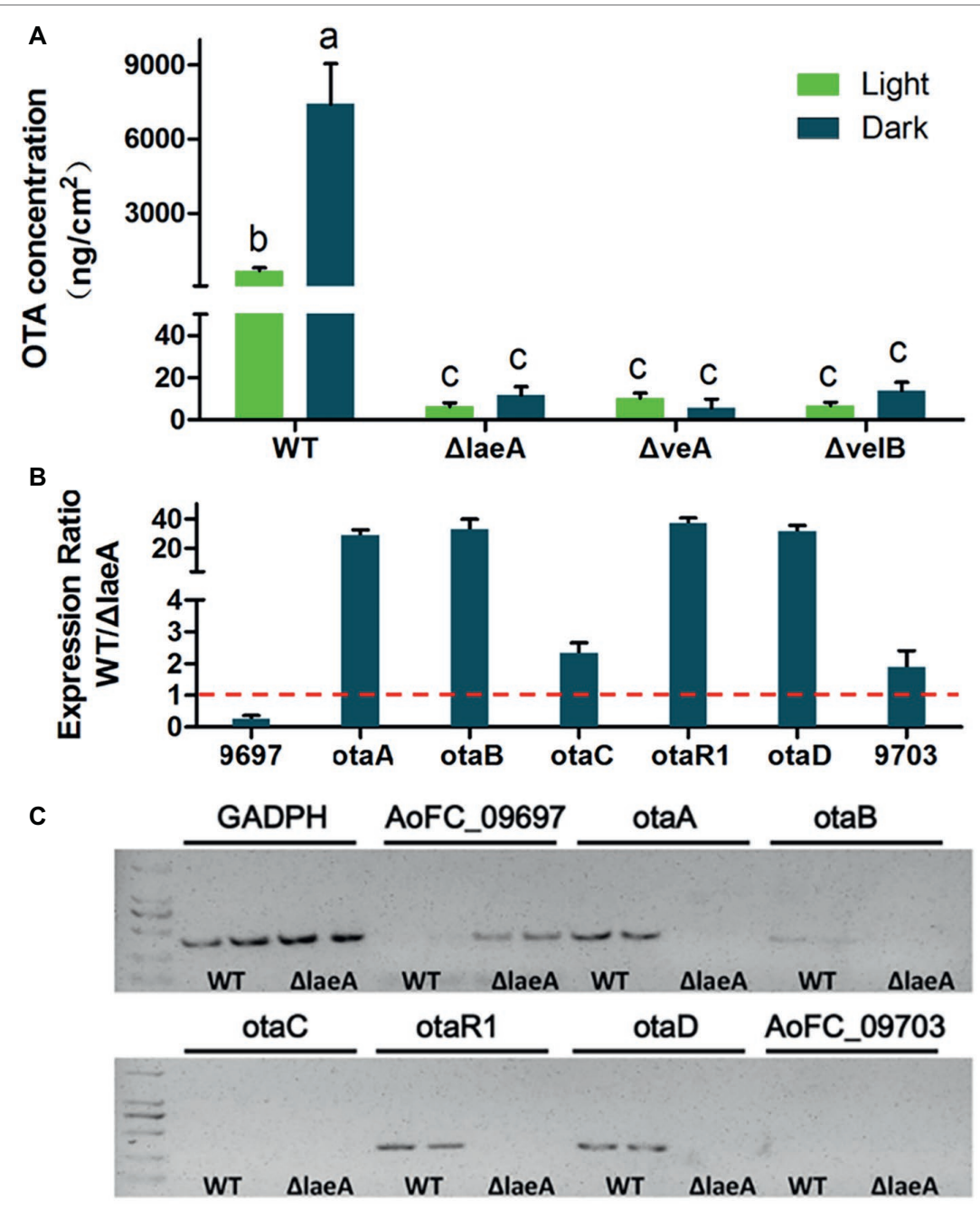

FIGURE 5 | OTA production in WT, $\Delta$ laeA, $\Delta$ veA, and $\Delta$ velB of $A$. ochraceus. (A) OTA concentration in WT, $\Delta$ laeA, $\Delta$ veA, and $\Delta$ velB under light and dark conditions. Different letters indicate a significant difference between the corresponding values $(p<0.05)$ with three biological replicates. (B) qRT-PCR was run to check the expression ratio of the genes which are involved OTA biosynthesis and as well as present inside and outside of OTA biosynthetic gene cluster in WT and compared to $\Delta$ laeA mutant. (C) RT-PCR amplification of the genes in and out OTA biosynthetic gene cluster.

to the OTA biosynthetic gene. The transcripts of the four OTA biosynthetic genes (otaA, otaB, otaD, and otaR1) were detected in WT by amplification by RT-PCR but not in $\triangle$ laeA. The otaC gene was not detected in WT because of its low level of expression (Figure 5C). These results were consistent with the production of OTA, which could be detected in WT and could not be detected in $\Delta$ laeA.

\section{Roles of LaeA, VeA, and VelB in Fungal Virulence}

The influence of LaeA, VeA, and VelB on the capacity of $A$. ochraceus to infect pears was ascertained. Lesion diameters were measured at 5 and 9 days after infection. After incubation for 5 days, lesions infected by all $A$. ochraceus strains were observed. Obviously, the lesions infected by $\Delta$ laeA, $\Delta$ veA, and $\Delta$ velB were repressed when compared with the lesions infected by WT (Figure 6A). Figure 6B demonstrated the significant difference in statistic level. After incubation for 9 days, the lesion infected by WT obviously increased. Lesions infected by $\Delta$ veA and $\Delta$ velB had little change compared with incubation for 5 days. This study illustrated that the loss of velvet proteins would weaken the infection ability of $A$. ochraceus on pear.

\section{LaeA Extensively Regulated Secondary Metabolism in A. ochraceus}

As earlier reported, the A. ochraceus genome contains 99 secondary metabolite biosynthetic gene clusters (Wang et al., 2018a,b). The expression level of backbone genes in secondary metabolites cluster were checked by qRT-PCR (Figure 7). About $66.1 \%$ of the backbone genes in the cluster were differentially expressed at $p<0.01$, and $81.6 \%$ of the differential expression genes were down-regulated in laeA deletion mutant. About $58.6 \%$ of the backbone genes' expression level were regulated at least two folds, among which $81.2 \%$ were down-regulated. 


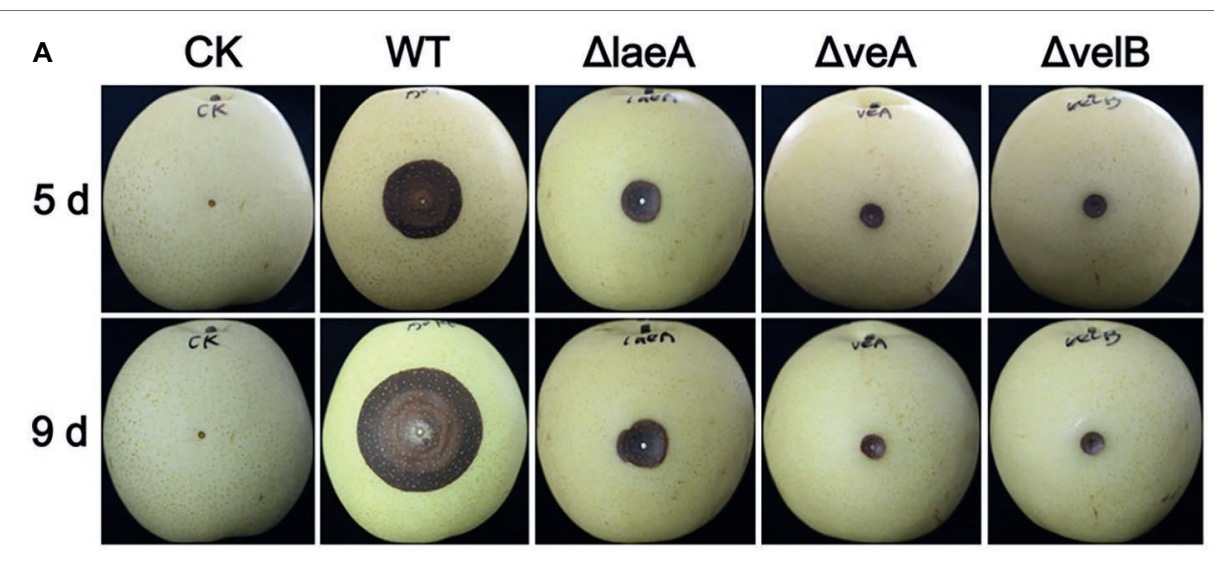

B

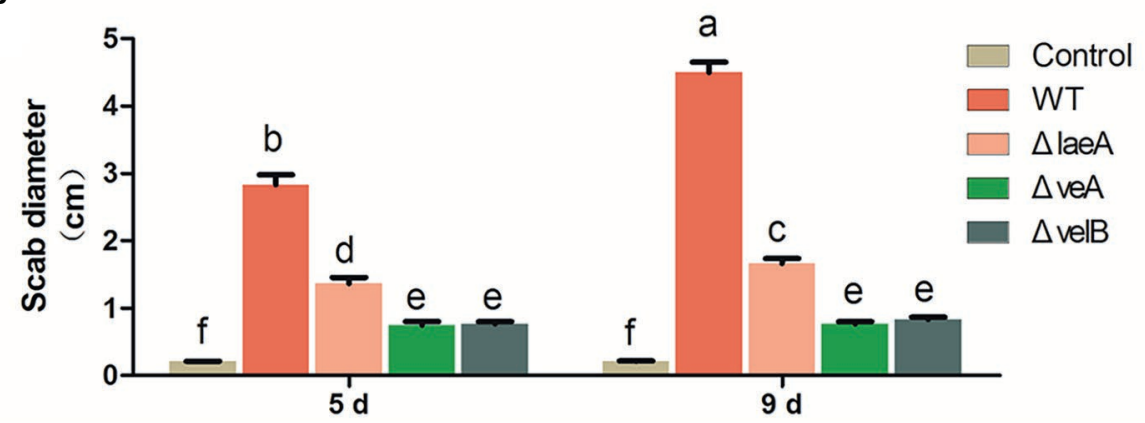

FIGURE 6 | Pathogenicity assay for WT and mutants of $A$. ochraceus on pears. (A) Pears infected by WT, $\Delta$ laeA, $\Delta$ veA, and $\Delta$ velB incubated at $28^{\circ} \mathrm{C}$ for 5 and 9 days under dark condition and photographed. (B) The scab diameters of pears measured using cross method. Different letters indicate a significant difference between the corresponding values $(p<0.05)$ with three biological replicates.
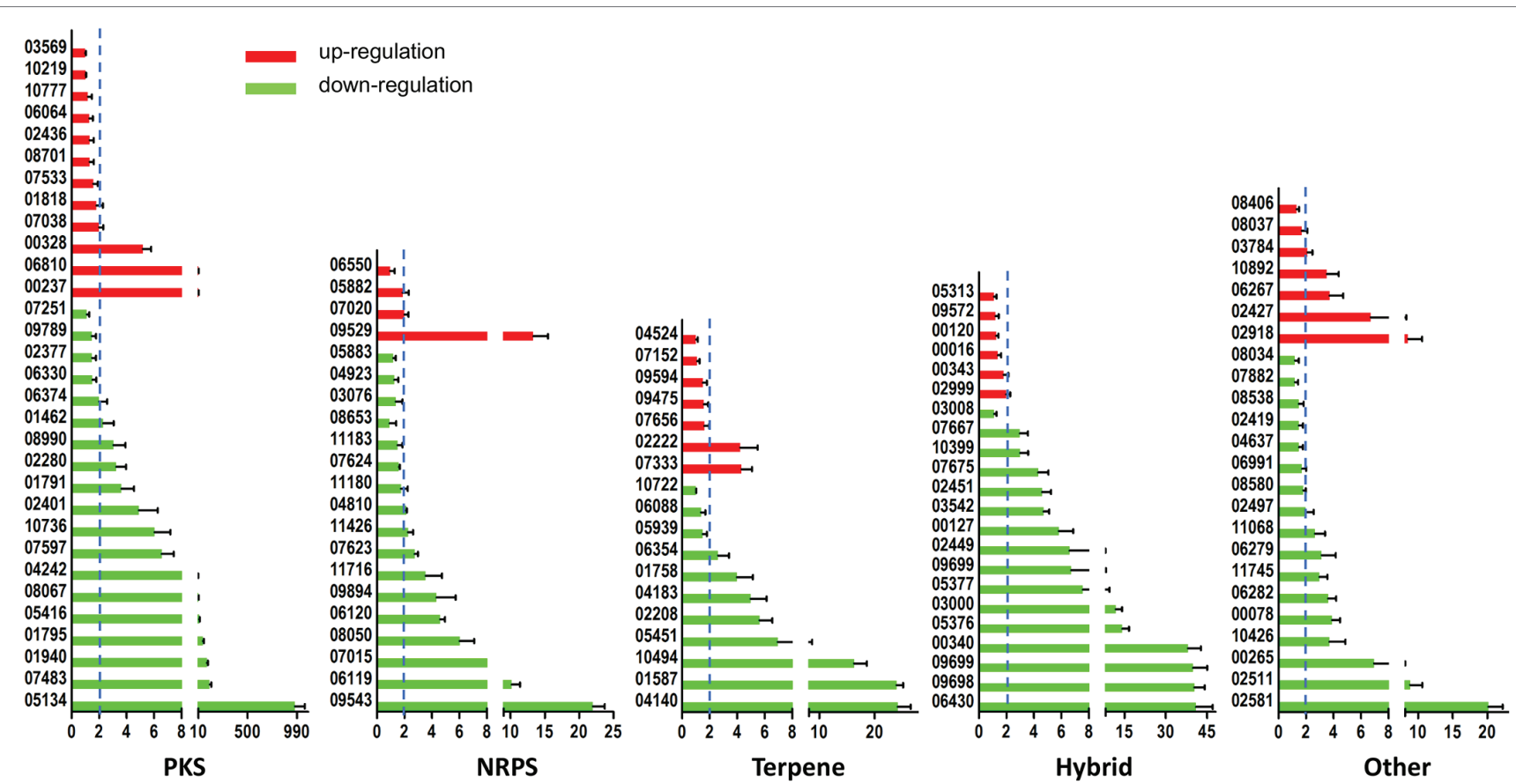

FIGURE 7 | LaeA influenced the expression level of secondary metabolite biosynthetic genes. Both WT and $\Delta$ laeA had three biological replicates. Y axes represented the backbone genes in PKS, NRPS, Terpene, Hybrid, and other gene clusters. X axes represented the expression ratio of genes expressed in WT compared to that expressed in $\Delta$ laeA. 
These results indicated that LaeA was essential for the expression of considerable part of secondary metabolite encoding genes.

\section{DISCUSSION}

OTA contamination of food, feed, and fruits is a significant health concern worldwide. A. ochraceus is the major producer of OTA, with a wide range of host. Furthermore, a number of secondary metabolites, such as circumdatin G and $\mathrm{H}$ (Dai et al., 2001; Lopez-Gresa et al., 2005), stephacidin A and B (Jingfang QianCutrone et al., 2002), Speramides A and B (Chang et al., 2016), and waspergillamide B (Frank et al., 2019), could be produced by $A$. ochraceus and researchers never give up to isolate new compounds from this fungus. However, the role of secondary metabolites except ochratoxins on health and virulence is unknown. And little is known about the genetic regulation of the lots of secondary metabolites including OTA biosynthesis process. Thus, deep inspection of the regulatory genes involved in metabolic pathways could provide a better understanding the mechanism of regulation of secondary metabolites.

In 2008, it was revealed that LaeA and two velvet families, VeA and VelB, confirmed a trimetirc complex that is essential to coordinate secondary metabolism and development in $A$. nidulans under dark condition (Bayram et al., 2008). VeA forms the light-responsive bridge that links VelB and LaeA. Three proteins were conserved in various fungi. In the WT of $A$. ochraceus, light cause a 50\% increase of conidiospore and a $92 \%$ reduction of OTA. It is found that OTA biosynthesis was reduced under light condition for other ochratoxingenic fungi such as $A$. carbonarius, $A$. niger, $P$. verrucosum, and $P$. nordicum (Schmidt-Heydt et al., 2010; Crespo-Sempere et al., 2013), indicated that the development and secondary metabolism was regulated by light condition and might be explained according to the role of velvet complex. Here, we are reporting first time the function of LaeA, VeA, and VelB in A. ochraceus, and also providing the vision on light regulating OTA biosynthesis mechanism.

Thus, we obtain the deletion mutants of laeA, veA, and $v e l B$ of $A$. ochraceus and compare their characteristic for development, OTA biosynthesis and fungal virulence on pears. Deletion of laeA led to the dramatic reduction of conidiaspore, and deletion of laeA, VeA, and VelB led to the slowing down of growth rate. The biosynthesis of OTA was strongly regulated by LaeA, VeA, and VelB, for the production of OTA was decreased by three order of magnitude in the deletion mutants. All the three proteins affected the pathogenicity of A. ochraceus on pears. However, we could not confirm whether pathogenicity be related to OTA biosynthesis. Some studies were reported to prove the role of mycotoxin in fungal virulence (Barad et al., 2014), whereas others not (Ballester et al., 2015). It is meaningful to in-depth study the relationship among development, OTA biosynthesis and fungal virulence of $A$. ochraceus for exploring strategies of OTA contamination.

The mechanism of LaeA playing its regulatory role is unclear until now, although a number of studies referring to various fungi focus on LaeA. Being a member of velvet complex is only one of the mechanisms. The S-adenosyl methionine-binding site contained in LaeA presumably indicates its methyltransferase activity. Additionally, it has been suggested that this protein has been linked to changes in chromatin structure because loss of LaeA leads to increased hetero-chromatin marks and its often precise regulation of secondary metabolites (Bok and Keller, 2016). In this study, we focused on the regulatory role of LaeA on secondary metabolite biosynthetic genes for its widely accepted function. About $66.7 \%$ backbone genes in NRPS cluster were significantly regulated by LaeA, among which about $85.7 \%$ of the genes were down-regulated. In addition to backbone genes in PKS, Terperne, hybrid, and other clusters, $66.1 \%$ of the genes were significantly regulated, and $81.6 \%$ of differential expression genes were downregulated (Figure 7). These data proved the role of LaeA in secondary metabolite biosynthesis regulation, and deletion of laeA repressed the expression of many compounds as reported previously (Bok and Keller, 2004; Perrin et al., 2007). Although the structure of compounds corresponding to each cluster was not clear, this study would gain insights to the link between compounds and biosynthetic gene clusters.

In conclusion, results from this study have provided some evidence that velvet complex proteins (LaeA, VeA, and VelB) play important roles in morphology development, OTA biosynthesis and fungal virulence in A. ochraceus. And we further demonstrated LaeA widely affect gene expression of A. ochraceus genome, with a focus on secondary metabolites. The down regulation effect of LaeA was more than up regulation effect in secondary metabolism. Given the strong effect of laeA, veA, and velB on OTA biosynthesis, these genes could be designed as target sites to develop new strategies for OTA control and prevention.

\section{DATA AVAILABILITY STATEMENT}

All datasets generated for this study are included in the article/Supplementary Material.

\section{AUTHOR CONTRIBUTIONS}

YL and GW designed the experiment. GW, YW, FL, EL, JM, $\mathrm{BY}$, and $\mathrm{CZ}$ performed the experiments. GW, LL, and $\mathrm{HZ}$ analyzed the data. GW wrote the manuscript.

\section{FUNDING}

The research was supported by the National Key R\&D Program of China (No. 2017YFC1600903), Beijing Natural Science Foundation (No. 6191001), and Central Public-interest Scientific Institution Basal Research Fund (No. S2019RCJC04 and S2019XTCG01).

\section{SUPPLEMENTARY MATERIAL}

The Supplementary Material for this article can be found online at: https://www.frontiersin.org/articles/10.3389/fmicb.2019.02759/ full\#supplementary-material 


\section{REFERENCES}

Abarca, M. L., Bragulat, M. R., Castella, G., and Cabanes, F. J. (2019). Impact of some environmental factors on growth and ochratoxin a production by Aspergillus niger and Aspergillus welwitschiae. Int. J. Food Microbiol. 291, 10-16. doi: 10.1016/j.ijfoodmicro.2018.11.001

Aziz, N. H., and Moussa, L. A. (1997). Influence of white light, near-UV irradiation and other environmental conditions on production of aflatoxin B1 by Aspergillus flavus and ochratoxin a by Aspergillus ochraceus. Nahrung 41, 150-154. doi: 10.1002/food.19970410307

Ballester, A. R., Marcet-Houben, M., Levin, E., Sela, N., Selma-Lazaro, C., Carmona, L., et al. (2015). Genome, transcriptome, and functional analyses of Penicillium expansum provide new insights into secondary metabolism and pathogenicity. Mol. Plant Microbe Interact. 28, 232-248. doi: 10.1094/ MPMI-09-14-0261-FI

Barad, S., Horowitz, S. B., Kobiler, I., Sherman, A., and Prusky, D. (2014). Accumulation of the mycotoxin patulin in the presence of gluconic acid contributes to pathogenicity of Penicillium expansum. Mol. Plant Microbe Interact. 27, 66-77. doi: 10.1094/MPMI-05-13-0138-R

Bayram, O., Krappmann, S., Ni, M., Bok, J. W., Helmstaedt, K., Valerius, O., et al. (2008). VelB/VeA/LaeA complex coordinates light signal with fungal development and secondary metabolism. Science 320, 1504-1506. doi: 10.1126/ science. 1155888

Bok, J. W., Balajee, S. A., Marr, K. A., Andes, D., Nielsen, K. F., Frisvad, J. C., et al. (2005). LaeA, a regulator of morphogenetic fungal virulence factors. Eukaryot. Cell 4, 1574-1582. doi: 10.1128/EC.4.9.1574-1582.2005

Bok, J. W., and Keller, N. P. (2004). LaeA, a regulator of secondary metabolism in Aspergillus spp. Eukaryot. Cell 3, 527-535. doi: 10.1128/EC.3.2. 527-535.2004

Bok, J. W., and Keller, N. P. (2016). "Insight into fungal secondary metabolism from ten years of LaeA research" in Biochemistry and molecular biology (the mycota). ed. D. Hoffmeister (Springer International Publishing Switzerland: Springer Press).

Chang, Y.-W., Yuan, C.-M., Zhang, J., Liu, S., Cao, P., Hua, H.-M., et al. (2016). Speramides A-B, two new prenylated indole alkaloids from the freshwaterderived fungus Aspergillus ochraceus KM007. Tetrahedron Lett. 57, 4952-4955. doi: 10.1016/j.tetlet.2016.09.071

Crespo-Sempere, A., Marin, S., Sanchis, V., and Ramos, A. J. (2013). VeA and LaeA transcriptional factors regulate ochratoxin a biosynthesis in Aspergillus carbonarius. Int. J. Food Microbiol. 166, 479-486. doi: 10.1016/j. ijfoodmicro.2013.07.027

Dai, J., Carte, B. K., Sidebottom, P. J., Sek Yew, A. L., Ng, S., Huang, Y., et al. (2001). Circumdatin G, a new alkaloid from the fungus Aspergillus ochraceus. J. Nat. Prod. 64, 125-126. doi: 10.1021/np000381u

Estiarte, N., Lawrence, C. B., Sanchis, V., Ramos, A. J., and Crespo-Sempere, A. (2016). LaeA and VeA are involved in growth morphology, asexual development, and mycotoxin production in Alternaria alternata. Int. J. Food Microbiol. 238, 153-164. doi: 10.1016/j.ijfoodmicro.2016.09.003

Frank, M., Özkaya, F., Müller, W., Hamacher, A., Kassack, M., Lin, W., et al. (2019). Cryptic secondary metabolites from the sponge-associated fungus Aspergillus ochraceus. Mar. Drugs 17:99. doi: 10.3390/md17020099

Gallo, A., Ferrara, M., and Perrone, G. (2017). Recent advances on the molecular aspects of ochratoxin a biosynthesis. Curr. Opin. Food Sci. 17, 49-56. doi: 10.1016/j.cofs.2017.09.011

Geisen, R., Schmidt-Heydt, M., Touhami, N., and Himmelsbach, A. (2018). New aspects of ochratoxin A and citrinin biosynthesis in Penicillium. Curr. Opin. Food Sci. 23, 23-31. doi: 10.1016/j.cofs.2018.04.001

IARC (1993). Some naturally occurring substances: food items and constituents, heterocyclic aromatic amines and mycotoxins. IARC Monogr. Eval. Carcinog. Risks Hum. 56, 489-521.

Jingfang Qian-Cutrone, S. H., Shu, Y.-Z., Vyas, D., Fairchild, C., Menendez, A., Krampitz, K., et al. (2002). Stephacidin A and B: two structurally novel, selective inhibitors of the testosterone-dependent prostate LNCaP cells. J. Am. Chem. Soc. 124, 14556-14557. doi: 10.1021/ja028538n

Kale, S. P., Milde, L., Trapp, M. K., Frisvad, J. C., Keller, N. P., and Bok, J. W. (2008). Requirement of LaeA for secondary metabolism and sclerotial production in Aspergillus flavus. Fungal Genet. Biol. 45, 1422-1429. doi: 10.1016/j.fgb.2008.06.009
Karimi-Aghcheh, R., Bok, J. W., Phatale, P. A., Smith, K. M., Baker, S. E., Lichius, A., et al. (2013). Functional analyses of Trichoderma reesei LAE1 reveal conserved and contrasting roles of this regulator. G3 3, 369-378. doi: $10.1534 / \mathrm{g} 3.112 .005140$

Lopez-Berges, M. S., Schafer, K., Hera, C., and Di Pietro, A. (2014). Combinatorial function of velvet and AreA in transcriptional regulation of nitrate utilization and secondary metabolism. Fungal Genet. Biol. 62, 78-84. doi: 10.1016/j. fgb.2013.11.002

Lopez-Gresa, M. P., Gonzalez, M. C., Primo, J., Moya, P., Romero, V., and Estornell, E. (2005). Circumdatin $\mathrm{H}$, a new inhibitor of mitochondrial NADH oxidase, from Aspergillus ochraceus. J. Antibiot. 58, 416-419. doi: 10.1038/ja.2005.54

Mantle, P. G. (2002). Risk assessment and the importance of ochratoxins. Int. Biodeterior. Biodegradation 50, 143-146. doi: 10.1016/S0964-8305(02)00079-3

Oda, K., Kobayashi, A., Ohashi, S., and Sano, M. (2011). Aspergillus oryzae laeA regulates kojic acid synthesis genes. Biosci. Biotechnol. Biochem. 95, 1832-1834. doi: 10.1271/bbb.110235

Perrin, R. M., Fedorova, N. D., Bok, J. W., Cramer, R. A., Wortman, J. R., Kim, H. S., et al. (2007). Transcriptional regulation of chemical diversity in Aspergillus fumigatus by LaeA. PLoS Pathog. 3:e50. doi: 10.1371/journal. ppat.0030050

Reverberi, M., Gazzetti, K., Punelli, F., Scarpari, M., Zjalic, S., Ricelli, A., et al. (2012). Aoyap1 regulates OTA synthesis by controlling cell redox balance in Aspergillus ochraceus. Appl. Microbiol. Biotechnol. 95, 1293-1304. doi: 10.1007/s00253-012-3985-4

Schmidt-Heydt, M., Bode, H., Raupp, F., and Geisen, R. (2010). Influence of light on ochratoxin biosynthesis by Penicillium. Mycotoxin Res. 26, 1-8. doi: 10.1007/s12550-009-0034-y

Schmidt-Heydt, M., Graf, E., Stoll, D., and Geisen, R. (2012). The biosynthesis of ochratoxin a by Penicillium as one mechanism for adaptation to $\mathrm{NaCl}$ rich foods. Food Microbiol. 29, 233-241. doi: 10.1016/j.fm.2011.08.003

Schmidt-Heydt, M., Rufer, C., Raupp, F., Bruchmann, A., Perrone, G., and Geisen, R. (2011). Influence of light on food relevant fungi with emphasis on ochratoxin producing species. Int. J. Food Microbiol. 145, 229-237. doi: 10.1016/j.ijfoodmicro.2010.12.022

Selouane, A., Bouya, D., Lebrihi, A., Decock, C., and Bouseta, A. (2009). Impact of some environmental factors on growth and production of ochratoxin A of/by Aspergillus tubingensis, A. niger, and A. carbonarius isolated from moroccan grapes. J. Microbiol. 47, 411-419. doi: 10.1007/s12275-008-0236-6

Taniwaki, M. H., Pitt, J. I., and Magan, N. (2018). Aspergillus species and mycotoxins: occurrence and importance in major food commodities. Curr. Opin. Food Sci. 23, 38-43. doi: 10.1016/j.cofs.2018.05.008

van der Merwe, K. J., Steyn, P. S., Fourie, L., Scott, D. B., and Theron, J. J. (1965). Ochratoxin A, a toxic metabolite produced by Aspergillus ochraceus Wilh. Nature 205, 1112-1113. doi: 10.1038/2051112a0

Veiga, T., Nijland, J. G., Driessen, A. J., Bovenberg, R. A., Touw, H., van den Berg, M. A., et al. (2012). Impact of velvet complex on transcriptome and penicillin $\mathrm{G}$ production in glucose-limited chemostat cultures of a betalactam high-producing Penicillium chrysogenum strain. OMICS 16, 320-333. doi: 10.1089/omi.2011.0153

Wang, G., Liu, Z., Lin, R., Li, E., Mao, Z., Ling, J., et al. (2016a). Biosynthesis of antibiotic leucinostatins in bio-control fungus Purpureocillium lilacinum and their inhibition on Phytophthora revealed by genome mining. PLoS Pathog. 12:e1005685. doi: 10.1371/journal.ppat.1005685

Wang, Y., Liu, F., Wang, L., Wang, Q., Selvaraj, J. N., Zhao, Y., et al. (2018a). pH-signaling transcription factor AopacC regulates Ochratoxin A biosynthesis in Aspergillus ochraceus. J. Agric. Food Chem. 66, 4394-4401. doi: 10.1021/ acs.jafc. 8 b00790

Wang, Y., Wang, L., Liu, F., Wang, Q., Selvaraj, J. N., Xing, F., et al. (2016b). Ochratoxin A producing fungi, biosynthetic pathway and regulatory mechanisms. Toxins 8. doi: 10.3390/toxins 8030083

Wang, L., Wang, Y., Wang, Q., Liu, F., Selvaraj, J. N., Liu, L., et al. (2015). Functional characterization of new polyketide synthase genes involved in Ochratoxin A biosynthesis in Aspergillus ochraceus fc-1. Toxins 7, 2723-2738. doi: $10.3390 /$ toxins 7082723

Wang, Y., Wang, L., Wu, F., Liu, F., Wang, Q., Zhang, X., et al. (2018b). A consensus Ochratoxin A biosynthetic pathway: insights from the genome sequence of Aspergillus ochraceus and a comparative genomic analysis. Appl. Environ. Microbiol. 84, pii: e01009-18. doi: 10.1128/AEM.01009-18 
William, E. H., and Hamilton, P. B. (1979). Mycotoxins - their biosynthesis in fungi: ochratoxins - metabolites of combined pathways. J. Food Prot. $41,815-820$.

Wu, D., Oide, S., Zhang, N., Choi, M. Y., and Turgeon, B. G. (2012). ChLae1 and ChVell regulate $\mathrm{T}$-toxin production, virulence, oxidative stress response, and development of the maize pathogen Cochliobolus heterostrophus. PLoS Pathog. 8:e1002542. doi: 10.1371/journal.ppat.1002542

Zhang, J., Chen, H., Sumarah, M. W., Gao, Q., Wang, D., and Zhang, Y. (2018). veA gene acts as a positive regulator of conidia production, Ochratoxin a biosynthesis, and oxidative stress tolerance in Aspergillus niger. J. Agric. Food Chem. 66, 13199-13208. doi: 10.1021/acs.jafc.8b04523
Conflict of Interest: The authors declare that the research was conducted in the absence of any commercial or financial relationships that could be construed as a potential conflict of interest.

Copyright (c) 2019 Wang, Zhang, Wang, Liu, Li, Ma, Yang, Zhang, Li and Liu. This is an open-access article distributed under the terms of the Creative Commons Attribution License (CC BY). The use, distribution or reproduction in other forums is permitted, provided the original author(s) and the copyright owner(s) are credited and that the original publication in this journal is cited, in accordance with accepted academic practice. No use, distribution or reproduction is permitted which does not comply with these terms. 